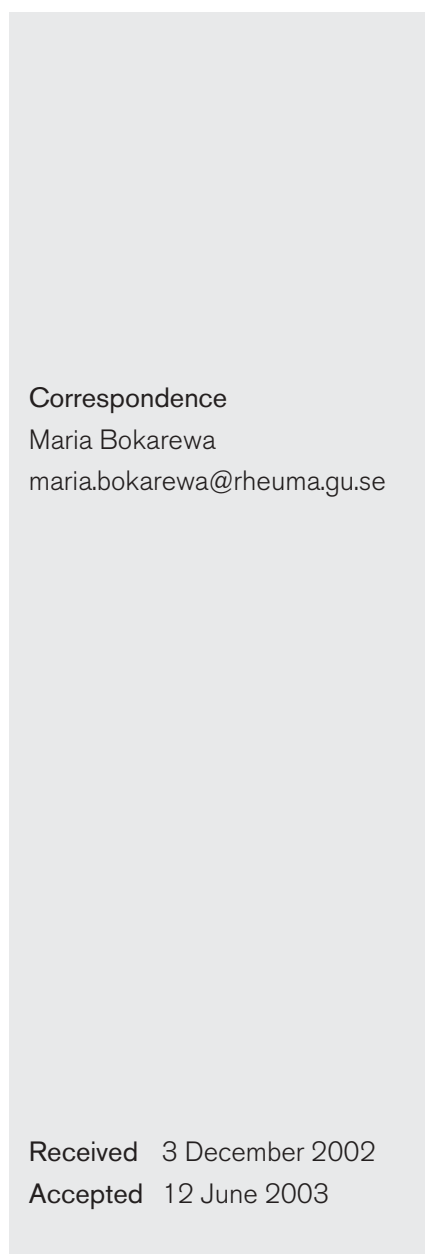

\title{
Fatal outcome of bacteraemic patients caused by infection with staphylokinase-deficient Staphylococcus aureus strains
}

\author{
Tao Jin, ${ }^{1}$ Maria Bokarewa, ${ }^{1}$ Lauren Mclntyre, ${ }^{2}$ Andrej Tarkowski, ${ }^{1}$ \\ G. Ralph Corey, ${ }^{3}$ L. Barth Reller ${ }^{3}$ and Vance G. Fowler, $\mathrm{Jr}^{3}$ \\ ${ }^{1}$ Department of Rheumatology and Inflammation Research, Sahlgrenska University Hospital, \\ Guldhedsgatan 10, S-413 46 Göteborg, Sweden \\ ${ }^{2}$ Department of Agronomy, Purdue University, West Lafayette, IN, USA \\ ${ }^{3}$ Division of Infectious Diseases, Duke University Medical Center, Durham, NC, USA
}

Staphylokinase (SAK) is a plasminogen-activator protein produced by Staphylococcus aureus. SAK production was evaluated in vitro in $S$. aureus isolates from the bloodstream of patients with lethal $(n=56)$ and non-lethal $(n=57)$ bacteraemia and from anterior nares of healthy subjects $(n=48)$. Most isolates (93/161) produced SAK, and $68 \%$ of SAK-producing isolates expressed both surface-bound and secreted types of SAK. SAK production was significantly less common among isolates from patients with lethal bacteraemia (39\%) than isolates from patients with non-lethal bacteraemia $(68 \%)$ or nasal carriage isolates $(67 \%)(P<0.01)$. After adjusting for infection with methicillin-resistant $S$. aureus and APACHE II score, patients infected with SAK-deficient isolates were 4.3 times more likely to have lethal bacteraemia than patients whose infecting isolate produced high levels of SAK $\left(\geqslant 5 \mu \mathrm{g} \mathrm{m}^{-1}\right)$, suggesting that in vitro SAK production was inversely associated with clinical outcome among patients with $S$. aureus bacteraemia. The high frequency of SAK production in nasal isolates and in cases with uncomplicated bacteraemia suggests that SAK may be one of the adaptive mechanisms of $S$. aureus symbiosis with the host.

\section{INTRODUCTION}

Staphylococcus aureus is a human pathogen that is a major cause of community- and hospital-acquired infections (Lowy, 1998). The pathogenicity of S. aureus is known to depend on a set of extracellular and cell wall-associated proteins that are not required for growth and cell division but are advantageous in particular environments (Foster \& Hook, 1998; Joh, 1999; Winzer \& Williams, 2001) and can influence the clinical severity of infection (Baba et al., 2002; Peacock et al., 2002; Gillet et al., 2002).

Several S. aureus proteins interact with host fibrinolysis. $S$. aureus interacts with host fibrinolysis by expressing plasminogen-binding moieties on the cell surface (Kuusela \& Saksela, 1990) and by producing a plasminogen activator, staphylokinase (SAK) (Collen, 1998). SAK is carried by a converting phage that inserts into the $\beta$-haemolysin structural gene. Expression of SAK is positively regulated by accessory gene regulator (Agr) and negatively regulated by

Abbreviations: MRSA, methicillin-resistant Staphylococcus aureus; SAK, staphylokinase. staphylococcal accessory gene regulator (Sar). The association between SAK expression and the outcome of S. aureus infections has not previously been evaluated.

In the present study, we assessed the pathogenic significance of SAK production for the invasive potential and outcome of staphylococcal infection using a collection of well-defined clinical isolates. Our results demonstrate a negative correlation between SAK production and the development of life-threatening complications and mortality during staphylococcal bacteraemia. In contrast, nasal isolates and strains of $S$. aureus giving rise to uncomplicated bacteraemia were more often SAK producers. These findings suggest that SAK is part of the adaptive mechanisms of $S$. aureus favourable for bacterial symbiosis with the host.

\section{METHODS}

Clinical characteristics of the patients. The study was approved by the Duke University Medical Center Institutional Review Board. Subjects permitting nasal swabbing provided written informed consent. Patient identification and selection methods have been described previously (Fowler et al., 2000). Briefly, daily reports were received from the microbiology laboratory about all patients at Duke University Medical Center with one or more blood cultures positive for S. aureus 
and clinical evidence of infection. The clinical charts of these patients were then reviewed within $36 \mathrm{~h}$ of detection of bacteraemia to confirm the presence of a clinical infection. Clinical data were collected at the time of patient hospitalization by investigators and entered into a computer database (Microsoft Access). The APACHE II score (Knaus et al., 1985) was evaluated for each patient on the day of the first blood culturing that yielded S. aureus. Exclusion criteria included S. aureus bacteraemia in patients who were outpatients or less than 18 years of age, as well as those with polymicrobial infection, those with neutropenia (absolute neutrophil count less than $1.0 \times 10^{9} \mathrm{l}^{-1}$ ) or those who died before evaluation by the investigators. Only $S$. aureus isolates from patients meeting study definitions for either invasive bacteraemia or uncomplicated bacteraemia (see below) were included in the current investigation

Study definitions. Specific clinical criteria were used to group bacterial isolates into three clinical categories: (i) lethal bacteraemia, (ii) nonlethal bacteraemia or (iii) nasal carriage isolates. Lethal bacteraemia isolates were obtained from the bloodstream of patients with S. aureus bacteraemia who died of their infection. Non-lethal isolates were obtained from the bloodstream of patients with clinically uncomplicated bacteraemia, as defined by the presence of all of the following features: (i) clinically significant intravascular catheter-associated S. aureus bacteraemia, (ii) no evidence of metastatic infection in internal organs, (iii) 14 days or less of intravenous antibiotic therapy and (iv) alive with no complications of infection 12 weeks after the initial positive blood culture. Nasal carriage isolates were obtained from the anterior nares of healthy non-medical university students by rotating a sterile Dacron fibre-tipped swab within both nostrils.

Isolation and characterization of $\mathbf{S}$. aureus strains. Blood culture isolates were identified as $S$. aureus and stored for future use, as described elsewhere (Fowler et al., 2000). Blood and nasal cultures were identified as containing $S$. aureus by subculturing on sheep blood agar, Gram staining the isolate and then performing the Staphaurex test (Murex Diagnostics), a latex agglutination assay that detects S. aureusspecific proteins. Isolates were kept frozen $\left(-70{ }^{\circ} \mathrm{C}\right)$ from the time of identification until they were analysed.

Culturing of S. aureus strains for SAK production. All assays were performed at the Department for Rheumatology and Inflammation Research, Göteborg University, Göteborg, Sweden, blinded to the source of the bacterial isolates. Prior to determination of SAK production, frozen $S$. aureus isolates were cultured on horse blood agar overnight and the bacteria within a single c.f.u. were used for further analyses.

One c.f.u. of each S. aureus strain was grown overnight in $2 \mathrm{ml}$ ToddHewitt broth (THB; Difco) at $37^{\circ} \mathrm{C}$. Staphylococci were subsequently harvested by centrifugation at $4000 \mathrm{~g}$ for $10 \mathrm{~min}\left(4^{\circ} \mathrm{C}\right)$. Supernatants were collected for further determination of secreted SAK activity. The bacterial pellet was assessed spectrophotometrically at $600 \mathrm{~nm}$ for bacterial density, washed with PBS (10 mM, pH 7.4) and used for evaluation of surface-bound SAK activity.

Staphylococci were resuspended in THB at $10^{9}$ c.f.u. $\mathrm{ml}^{-1}$ and incubated for $4 \mathrm{~h}$ at $37^{\circ} \mathrm{C}$ with $20 \mu \mathrm{g}$ human Glu-plasminogen $\mathrm{ml}^{-1}$ (Biopool). Excess plasminogen was removed by washing twice with PBS. The bacterial pellet was resuspended in Tris/ $\mathrm{HCl}$ buffer $(50 \mathrm{mM}$, $\mathrm{pH} 7 \cdot 4$ ) containing plasmin substrate (see below) and incubated for another $30 \mathrm{~min}$ to allow SAK-dependent activation of plasminogen attached to the bacterial surface.

Determination of SAK activity. SAK activity of bacterial supernatants was determined by incubation with $0 \cdot 2 \mu \mathrm{M}$ human Glu-plasminogen (Biopool) in Tris/HCl buffer (50 mM, pH 7.4) to allow SAK-dependent conversion of plasminogen to plasmin. Plasmin formation was eval- uated by hydrolysis of $0 \cdot 4 \mu \mathrm{M} H$-D-Val-Leu-Lys paranitroanilide (S-2251; Chromogenix), a specific plasmin substrate. The $A_{405}$ was compared with values obtained from standard dilutions of recombinant SAK (sakSTAR, a kind gift of Dr Desire Collen, Center for Thrombosis and Vascular Research, Leuven, Belgium). The absorbance reading for surface-bound SAK was measured after $30 \mathrm{~min}$ and for secreted SAK after $2 \mathrm{~h}$ of incubation with plasminogen. The incubation time was adjusted to give similar sensitivity in the assays, allowing the detection of $\geqslant 0.3 \mu \mathrm{g} \mathrm{SAK} \mathrm{ml}{ }^{-1}$. Samples with absorbances below this level were considered negative. SAK levels ranging between 0.3 and $5 \mu \mathrm{g} \mathrm{ml}^{-1}$ were arbitrarily assessed as low production and above $5 \mu \mathrm{g} \mathrm{ml}^{-1}$ as high production. S. aureus strain Newman was used throughout as a positive control.

Statistical methods. The type of SAK production was assessed as none (i.e. below the detection level), surface-bound SAK alone or a combination of surface/secreted SAK. We looked at SAK production in a variety of ways. Our initial comparison of SAK production for both surface and secreted SAK was conducted by comparing the absorbances for the three clinical groups using Wilcoxon's rank sum test. Following this, the frequency of SAK-producing $S$. aureus isolates within the groups was assessed by pairwise comparison. The comparisons were performed between the patients and controls, invasive cases and uncomplicated cases and uncomplicated cases and controls. For each of these comparisons, a $\chi^{2}$ test was used.

Logistic regression analysis was also performed using case status (invasive or uncomplicated bacteraemia) as the outcome and secreted SAK as an independent variable. Because the proportionality assumption was not met, secreted SAK was transformed into an indicator variable with three levels to fit the logistic model: $<0 \cdot 3 \mu \mathrm{g} \mathrm{ml}^{-1}$ (none), $0 \cdot 3-5 \mu \mathrm{g} \mathrm{ml}^{-1}$ (low), $>5 \mu \mathrm{g} \mathrm{ml}^{-1}$ (high). To examine whether secreted SAK was a predictor of case status, a logistic model with case status as the outcome and secreted SAK was fitted. Furthermore, to determine whether the association between secreted SAK and outcome was independent of known risk factors for poor outcome, a logistic model with case status as the outcome and race, sex, APACHE II score, presence of methicillin-resistant S. aureus (MRSA) and secreted SAK was fitted. Backward selection was used to eliminate variables.

For the examination of surface SAK, a logistic model with case status as the outcome and a binary indicator of surface SAK as the independent variable was fitted. As with secreted SAK, a multivariate logistic model with case status as the outcome and race, sex, APACHE II score, presence of MRSA and secreted SAK was fitted and backward selection was used to eliminate variables.

\section{RESULTS}

\section{Clinical and demographic characteristics of patient groups}

Patients with complicated bacteraemia leading to death were more often over 65 years of age, more frequently females, more frequently infected with MRSA strains and had significantly higher APACHE II scores (Table 1).

Among the 56 patients with invasive $S$. aureus bacteraemia, the following complications were identified: infective endocarditis (27 patients, $48 \%$ ), deep tissue abscesses (11, $20 \%)$, meningitis $(6,11 \%)$, septic arthritis $(6,11 \%)$, empyema (5, 9\%), epidural abscess (5, 9\%), septic thrombophlebitis $(4,7 \%)$, vertebral osteomyelitis $(3,5 \%)$, pacemaker pocket infection $(2,4 \%)$ and mycotic aneurysm (1, $2 \%)$. According to study definitions, all patients with 
Table 1. Demographic characteristics of patients with uncomplicated $S$. aureus bacteraemia $(n=57)$ and with invasive $S$. aureus infection $(n=56)$

\begin{tabular}{|lccc|}
\hline Characteristic & $\begin{array}{c}\text { Uncomplicated } \\
\text { S. aureus } \\
\text { bacteraemia }\end{array}$ & $\begin{array}{c}\text { Invasive } \\
\text { S. aureus } \\
\text { bacteraemia }\end{array}$ & P value \\
\hline Age $>$ 65 years & $17(30 \%)$ & $33(59 \%)$ & $0 \cdot 0018$ \\
Male sex & $37(65 \%)$ & $26(46 \%)$ & $0 \cdot 0479$ \\
White race & $41(72 \%)$ & $35(63 \%)$ & $0 \cdot 3475$ \\
MRSA strains & $14(25 \%)$ & $34(61 \%)$ & $0 \cdot 0001$ \\
APACHE II score: & & & \\
$\quad<10$ & $25(43 \%)$ & $3(5 \%)$ & $0 \cdot 0001$ \\
$10-16$ & $17(30 \%)$ & $11(19 \%)$ & NS \\
$16-21$ & $11(19 \%)$ & $15(27 \%)$ & NS \\
$>21$ & $4(7 \%)$ & $27(48 \%)$ & $0 \cdot 0001$ \\
\hline
\end{tabular}

NS, Not significant.

invasive S. aureus bacteraemia died of their infection. All patients with uncomplicated bacteraemia had intravascular catheter-associated $S$. aureus bacteraemia, received 14 days or less of intravenous antibiotics and had no complications attributable to $S$. aureus at the time of the initial bacteraemia or at 12-week follow-up. Nasal strains were obtained from non-medical university students in their second decade of life.

\section{SAK production within the tested clinical isolates}

SAK production was detected in 93 of 161 (58\%) S. aureus strains tested (Table 2). This SAK production consisted of SAK being attached to the bacterial surface, referred to as surface-bound, and SAK being released into the fluid phase. Isolates with detectable surface-bound SAK also produced soluble SAK (Spearman's correlation coefficient for surfacebound and soluble SAK, $r=0 \cdot 71)$. Discordance between SAK production was observed in only $18 \%(29 / 161)$ of $S$. aureus isolates, equally often in the patient and in the control groups. In all these discordant cases, isolated surfacebound SAK expression was present.

\section{Relationship between SAK production and invasive potential and outcome of $\boldsymbol{S}$. aureus infection}

SAK production was significantly less common in isolates from patients with lethal bacteraemia (invasive bacteraemia isolates) than in either non-lethal (uncomplicated) bacteraemia or nasal-carriage isolates (invasive bacteraemia isolates versus uncomplicated and nasal-colonizing isolates; $\chi^{2}$ exact $P=0.018$ ) (Table 2). In addition, the magnitude of SAK production differed among the three groups. High levels $\left(>5 \mu \mathrm{g} \mathrm{ml}^{-1}\right)$ of SAK production were significantly more common among isolates from either non-lethal bacteraemia (uncomplicated bacteraemia isolates) $(23 / 57,40.4 \%)$ or nasal-carriage subjects (nasal-colonizing control isolates) $(20 / 48,41.7 \%)$ than isolates from patients with lethal bacteraemia (invasive bacteraemia isolates) $(10 / 56,17.9 \%)$ $(P=0 \cdot 002)$.

To evaluate further whether SAK production was associated with patient outcome (e.g. lethal versus non-lethal bacteraemia), a logistic regression model with patient outcome as the dependent variable and secreted, surface-bound and combined (secreted plus surface-bound) SAK was estimated. All three indicators of SAK production were associated significantly with outcome. In all three cases, the absence of SAK production was associated with lethal bacteraemia [secreted SAK (none versus high); odds ratio $(\mathrm{OR})=3.07$, $95 \%$ confidence interval $(\mathrm{CI})=1 \cdot 27-7 \cdot 40, P=0 \cdot 036$; surface-bound SAK, OR $=3 \cdot 09 ; 95 \% \mathrm{CI}=1 \cdot 43-6 \cdot 67, P=$ 0.004; combined surface-bound or secreted SAK, $\mathrm{OR}=3 \cdot 35 ; 95 \% \mathrm{CI}=1 \cdot 54-7 \cdot 26, P=0 \cdot 002]$.

In order to determine whether the effect of SAK was independently associated with patient outcome, a multivariate logistic regression model was developed with patient outcome as the dependent variable and race, sex, APACHE II score, infection with MRSA and secreted SAK as independent variables. Secreted SAK and surface-bound SAK were modelled separately and then combined. In all models, backward selection eliminated both race and sex from the model. After adjusting for APACHE II score and infection with MRSA, secreted SAK was significantly associated with patient outcome $[\mathrm{OR}$ (none versus high) $=4 \cdot 39,95 \% \mathrm{CI}=1 \cdot 21-$ 15.91, $P=0 \cdot 046]$. Thus, after adjustment for severity of

Table 2. SAK production in patients with uncomplicated $S$. aureus bacteraemia $(n=57)$ and invasive $S$. aureus infection $(n=56)$ and healthy nasal carriers $(n=48)$

\begin{tabular}{|lccc|}
\hline Type of SAK production & Nasal S. aureus & Uncomplicated S. aureus & Invasive S. aureus \\
\hline Secreted only & 0 & $1(1 \cdot 7 \%)$ & 0 \\
Surface only & $11(22 \cdot 9 \%)$ & $12(21 \cdot 1 \%)$ & $6(10 \cdot 7 \%)$ \\
Combined (surface/ & $21(43.8 \%)$ & $26(45 \cdot 6 \%)$ & $16(28 \cdot 6 \%)$ \\
secreted) & 1 & 3 & 6 \\
Low $\left(0 \cdot 3-5 \mu \mathrm{g} \mathrm{ml}^{-1}\right)$ & 20 & 23 & 10 \\
High $\left(>5 \mu \mathrm{gl}^{-1}\right)$ & $16(33.3 \%)$ & $18(31.6 \%)$ & $34(60 \cdot 7 \%)$ \\
None & & & \\
\hline
\end{tabular}


illness, comorbid conditions and infection with MRSA, individuals infected with non-SAK-producing S. aureus isolates were $4 \cdot 3$ times more likely to have lethal bacteraemia compared with individuals infected with $S$. aureus secreting high levels of SAK $\left(>5 \mu \mathrm{g} \mathrm{ml}^{-1}\right)$. By contrast, the presence of combined surface-bound and secreted SAK production was eliminated from a multivariate model containing APACHE II score and infection with MRSA $(\mathrm{OR}=2 \cdot 65, P=0 \cdot 080)$.

\section{DISCUSSION}

The findings of the present study demonstrate an association between in vitro SAK production among S. aureus bloodstream isolates and the clinical outcome of patients from whom these isolates were obtained. After adjusting for confounding host and pathogen-specific characteristics, patients with $S$. aureus bacteraemia due to non-SAK-producing isolates were significantly more likely to die of their infection than patients with bacteraemia due to $S$. aureus isolates that produced SAK. This inverse correlation between SAK production and $S$. aureus mortality has interesting implications for our understanding of the host-pathogen interaction.

Lack of SAK production in staphylococci invading internal organs is unexpected, especially in view of a previously suggested proteolytic mechanism of harbouring of $S$. aureus in tissues (Lahteenmaki et al., 2001; Christner \& Boyle, 1996). On the other hand, expression of SAK is followed by excessive plasmin formation. Plasmin-mediated degradation of extracellular matrix deprives bacteria of the necessary adherent surface (Foster \& Hook, 1998; Josefsson et al., 2001) and, thereby, decreases their invasive pathogenic potential. The high frequency of SAK production in nasal isolates suggests that production of SAK is an adaptive bacterial mechanism favourable for its symbiosis with the host. Expression of SAK may be an important property for $S$. aureus colonization of mucosal tissue. Nasal carriage of $S$. aureus is considered to be one of the major risk factors for a generalized staphylococcal infection (Harbarth et al., 2000; von Eiff et al., 2001). We observed that production of SAK is a feature that differentiates nasal isolates from staphylococci that invade internal organs. We have shown recently that SAK interacts with and neutralizes bactericidal peptides from human azurophilic granules (T. Jin, J. Mitchell, J. Higgins, T. Foster, A. Tarkowski and M. Bokarewa, submitted for publication), protecting $S$. aureus from the host innate immune system and facilitating bacterial survival.

There were several limitations to this investigation. It is possible that investigator awareness of the clinical source of infection may have biased the results. However, all isolations were performed at a separate site by investigators blinded to all clinical data. To guard further against analytical bias, all statistical investigations were also performed at a site separate from the source of the clinical isolates. Patient or bacterial characteristics other than SAK production may also have confounded these results. Despite adjustment of the patient groups for severity of illness and infection with
MRSA, we could not exclude the possibility that these two factors made a major contribution to the patient outcome of septicaemia.

The SAK gene-containing phage is inserted into the $\beta$-toxin gene, causing the loss of $\beta$-haemolysin expression. Thus, the increased mortality of bacteraemia with SAK-deficient isolates may be partly due to $\beta$-toxin production by these isolates. This suggestion seems less probable in view of recent studies that have shown a negligible impact of $\beta$-haemolysin on the virulence of staphylococcal strains (Nilsson et al., 1999; Dajcs et al., 2002). Additionally, we cannot exclude the possibility of a 'hitchhiker effect', in which the gene for SAK production is near an unknown gene that results in the observed differences.

Our study suggests an association between reduced SAK production in vitro among $S$. aureus bloodstream isolates and a worse overall clinical outcome in patients with $S$. aureus bacteraemia, indicating that important phenotypic differences exist in S. aureus isolates from different clinical settings. Keeping in mind that infection often occurs with one's own strains, determination of SAK production is a valuable factor to predict the potential risk of invasive infection among nasal staphylococcal carriers. Future investigations are necessary in order to validate this observation and to determine whether such clinically relevant differences in pathogen phenotype are due to selective expression in response to differing conditions confronting the infecting isolate or to constitutive differences in the bacterial genotype.

\section{ACKNOWLEDGEMENTS}

This work has been supported financially by the Göteborg Medical Society, the Swedish Association against Rheumatism, King Gustaf V's Foundation, the Swedish Medical Research Council, Nanna Svartz' Foundation, Börje Dahlin's Foundation, the Swedish National Inflammation Network and the National Institute of Health (AI-01647 to V.G. F.), the Purdue University Agricultural Experiment Station and the University of Göteborg.

\section{REFERENCES}

Baba, T., Takeuchi, F., Kuroda, M. \& 11 other authors (2002). Genome and virulence determinants of high virulence community-acquired MRSA. Lancet 359, 1819-1827.

Christner, R. B. \& Boyle, M. D. (1996). Role of staphylokinase in the acquisition of plasmin(ogen)-dependent enzymatic activity by staphylococci. J Infect Dis 173, 104-112.

Collen, D. (1998). Staphylokinase: a potent, uniquely fibrin-selective thrombolytic agent. Nat Med 4, 279-284.

Dajcs, J. J., Thibodeaux, B. A., Girgis, D. O. \& O'Callaghan, R. J. (2002). Corneal virulence of Staphylococcus aureus in an experimental model of keratitis. DNA Cell Biol 21, 375-382.

Foster, T. J. \& Hook, M. (1998). Surface protein adhesins of Staphylococcus aureus. Trends Microbiol 6, 484-488.

Fowler, V. G., Jr, McIntyre, L. M., Yeaman, M. R., Peterson, G. E., Barth Reller, L., Corey, G. R., Wray, D. \& Bayer, A. S. (2000). In vitro resistance to thrombin-induced platelet microbicidal protein in isolates of Staphylococcus aureus from endocarditis patients correlates with an intravascular device source. J Infect Dis 182, 1251-1254. 
Gillet, Y., Issartel, B., Vanhems, P. \& 11 other authors (2002). Association between Staphylococcus aureus strains carrying gene for Panton-Valentine leukocidin and highly lethal necrotising pneumonia in young immunocompetent patients. Lancet 359, 753-759.

Harbarth, S., Liassine, N., Dharan, S., Herrault, P., Auckenthaler, R. \& Pittet, D. (2000). Risk factors for persistent carriage of methicillinresistant Staphylococcus aureus. Clin Infect Dis 31, 1380-1385.

Joh, D., Wann, E. R., Kreikemeyer, B., Speziale, P. \& Hook, M. (1999). Role of fibronectin-binding MSCRAMMs in bacterial adherence and entry into mammalian cells. Matrix Biol 18, 211-223.

Josefsson, E., Hartford, O., O’Brien, L., Patti, J. M. \& Foster, T. (2001). Protection against experimental Staphylococcus aureus arthritis by vaccination with clumping factor $\mathrm{A}$, a novel virulence determinant. J Infect Dis 184, 1572-1580.

Knaus, W. A., Draper, E. A., Wagner, D. P. \& Zimmerman, J. E. (1985). APACHE II: a severity of disease classification system. Crit Care Med 13, $818-829$.

Kuusela, P. \& Saksela, O. (1990). Binding and activation of plasminogen at the surface of Staphylococcus aureus. Increase in affinity after conversion to the Lys form of the ligand. Eur J Biochem 193, 759-765.
Lahteenmaki, K., Kuusela, P. \& Korhonen, T. K. (2001). Bacterial plasminogen activators and receptors. FEMS Microbiol Rev 25, $531-552$.

Lowy, F. D. (1998). Staphylococcus aureus infections. N Engl J Med 339, $520-532$

Nilsson, I. M., Hartford, O., Foster, T. \& Tarkowski, A. (1999). Alphatoxin and gamma-toxin jointly promote Staphylococcus aureus virulence in murine septic arthritis. Infect Immun 67, 1045-1049.

Peacock, S. J., Moore, C. E., Justice, A., Kantzanou, M., Story, L., Mackie, K., O'Neill, G. \& Day, N. P. (2002). Virulent combinations of adhesin and toxin genes in natural populations of Staphylococcus aureus. Infect Immun 70, 4987-4996.

von Eiff, C., Becker, K., Machka, K., Stammer, H. \& Peters, G. (2001). Nasal carriage as a source of Staphylococcus aureus bacteremia. Study Group. N Engl J Med 344, 11-16.

Winzer, K. \& Williams, P. (2001). Quorum sensing and the regulation of virulence gene expression in pathogenic bacteria. Int J Med Microbiol 291, 131-143. 